\title{
Development of Ultra-Fast Silicon Detectors for 4D tracking
}

A. Staiano, ${ }^{a}, 1$ R. Arcidiacono,${ }^{e}$ M. Boscardin, ${ }^{c}$ G.F. Dalla Betta, ${ }^{b}$ N. Cartiglia, ${ }^{a}$ F. Cenna, ${ }^{a, d}$ M. Ferrero, ${ }^{a}$ F. Ficorella,${ }^{c}$ M. Mandurrino, ${ }^{a}$ M. Obertino, ${ }^{a, d}$ L. Pancheri, ${ }^{b}$ G. Paternoster ${ }^{c}$ and V. Sola ${ }^{a, d}$

${ }^{a}$ Istituto Nazionale di Fisica Nucleare, Sezione di Torino, via P. Giuria 1, Torino, Italy

${ }^{b}$ Dipartimento di Fisica, Università di Trento, via Sommarive 14, Povo (Trento), Italy

${ }^{c}$ Fondazione Bruno Kessler, Center for Materials and Microsystems, via Sommarive 18, Povo (Trento), Italy

${ }^{d}$ Dipartimento di Fisica, Università di Torino, via P. Giuria 1, Torino, Italy

${ }^{e}$ Dipartimento di Scienze Farmaceutiche, Università del Piemonte Orientale, Largo Donegani 2/3, Novara, Italy

E-mail: amedeo.staiano@to.infn.it

AвstRact: In this contribution we review the progress towards the development of a novel type of silicon detectors suited for tracking with a picosecond timing resolution, the so called Ultra-Fast Silicon Detectors.

The goal is to create a new family of particle detectors merging excellent position and timing resolution with $\mathrm{GHz}$ counting capabilities, very low material budget, radiation resistance, fine granularity, low power, insensitivity to magnetic field, and affordability. We aim to achieve concurrent precisions of $\sim 10 \mathrm{ps}$ and $\sim 10 \mu \mathrm{m}$ with a $50 \mu \mathrm{m}$ thick sensor.

Ultra-Fast Silicon Detectors are based on the concept of Low-Gain Avalanche Detectors, which are silicon detectors with an internal multiplication mechanism so that they generate a signal which is factor $\sim 10$ larger than standard silicon detectors. The basic design of UFSD consists of a thin silicon sensor with moderate internal gain and pixelated electrodes coupled to full custom VLSI chip.

An overview of test beam data on time resolution and the impact on this measurement of radiation doses at the level of those expected at HL-LHC is presented.

\footnotetext{
${ }^{1}$ Corresponding author.
} 
First I-V and C-V measurements on a new FBK sensor production of UFSD, $50 \mu \mathrm{m}$ thick, with $\mathrm{B}$ and $\mathrm{Ga}$, activated at two diffusion temperatures, with and without $\mathrm{C}$ co-implantation (in Low and High concentrations), and with different effective doping concentrations in the Gain layer, are shown.

Perspectives on current use of UFSD in HEP experiments (UFSD detectors have been installed in the CMS-TOTEM Precision Protons Spectrometer for the forward physics tracking, and are currently taking data) and proposed applications for a MIP timing layer in the HL-LHC upgrade are briefly discussed.

KeYwords: Charge transport and multiplication in solid media; Detector modelling and simulations II (electric fields, charge transport, multiplication and induction, pulse formation, electron emission, etc); Particle tracking detectors (Solid-state detectors); Radiation-hard detectors 


\section{Contents}

1 Time measurement in tracking at high luminosity High Energy Physics experiments 1

2 Time resolution in silicon detectors $\quad 2$

2.1 Front-end and read-out electronics 3

2.2 Beam test results 4

3 UFSD radiation tolerance $\quad 4$

4 New FBK production $\quad 7$

5 Perspectives on UFSD $\quad 8$

6 Conclusions 9

\section{Time measurement in tracking at high luminosity High Energy Physics experiments}

Time, as a $4^{\text {th }}$ dimension in High Energy Physics tracking, will be a crucial measurement in the harsh environment of high luminosity collider experiments (see figure 1). Silicon detectors have proven in the last decades to be an excellent choice in large scale ( $\gtrsim 10 \mathrm{M}$ channels) tracking detectors, given the high spatial resolution $(\sim 10 \mu \mathrm{m})$, the radiation tolerance, the fabrication process stability and the cost. The high granularity and resolution of standard n-p junctions however is not matched by their time resolution. Currently the best time resolution performance on a running experiment comes from the Gigatracker detector of NA62 at CERN [1], with $\sigma_{t} \simeq 135$ ps on $300 \times 300 \mu \mathrm{m}$ pixels.

The possibility of conjugating the spatial resolution of high granularity silicon detectors with good time resolution would be of great benefit to the tracking algorithms, which could be rewritten taking into account the time evolution of each hit associated to a track, thus suppressing wrongly associated hits. Moreover time associated to the primary vertex can be used to improve event reconstruction, in those cases in which two events superimpose given the proximity of the two primary vertices (as in the event of figure 1). A high resolution time stamp will help not only at the reconstruction level but also at the trigger level, associating the event to the correct trigger algoritm (figure 2).

Tracking in high density events will be crucial in the next generations of high luminosity high energy physics experiments. HL-LHC [2] is expected to reach, at full luminosity, rates of up to 200 vertices/events, with a linear density of $\sim 1.8$ events $/ \mathrm{mm}$. With a time vertex distribution of $<\mathrm{t}_{\text {vertex }}>_{\mathrm{RMS}} \simeq 200 \mathrm{ps}$, and a vertex separation resolution of $\sim 300 \mu \mathrm{m}$, the fraction of unresolved events will be $\sim 15 \%$. 4D tracking, with timing resolutions of $\sim 30 \mathrm{ps}$ is expected to reduce by a factor of $\sim 5$ the number of pileup tracks per primary vertex, at the maximum luminosity rate of 200 PU events/beam crossing [3]. 


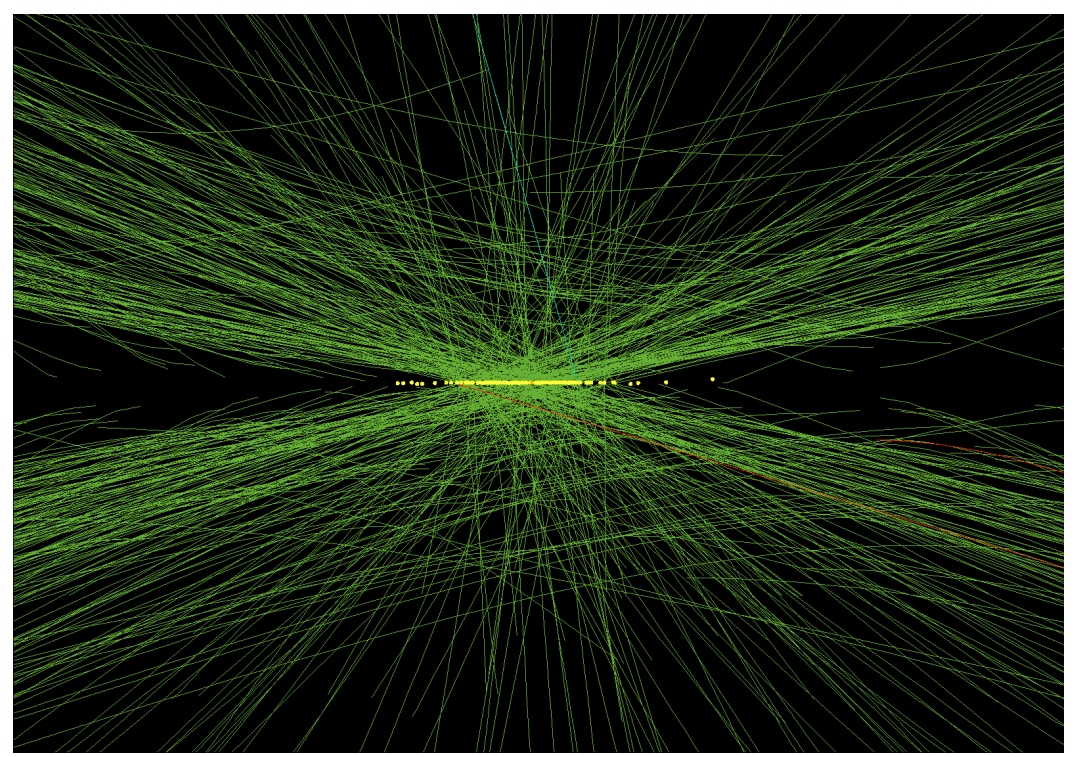

Figure 1. 78 pileup events reconstructed in a single bunch crossing at the CERN experiment CMS.

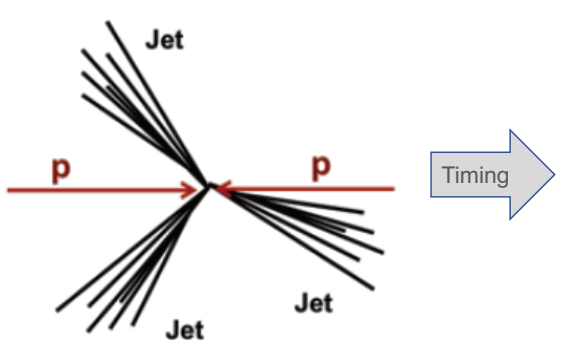

3-Jet event?

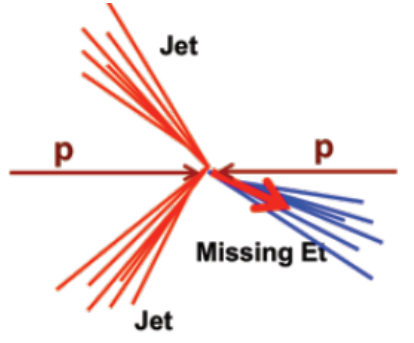

2-Jet and Missing Et event

Figure 2. Timing information resolves 2-jets + missing $\mathrm{E}_{T}$ superimposed to a single jet event.

\section{Time resolution in silicon detectors}

The time of crossing of a particle through the sensitive layer of a particle detector is given by the time at which the amplified signal crosses a given threshold $V_{\text {th }}$. The error on this quantity is the time resolution and it is the convolution of several contributions:

$$
\sigma_{t}^{2}=\sigma_{\text {Jitter }}^{2}+\sigma_{\text {Time Walk }}^{2}+\sigma_{\text {Landau Noise }}^{2}+\sigma_{\text {Distortion }}^{2}+\sigma_{\text {TDC }}^{2}
$$

The term $\sigma_{\mathrm{Jitter}}$ is proportional to the signal noise and inversely proportional to the signal slew rate, $\sigma_{\mathrm{Jitter}} \simeq N /(d V / d t)=t_{\text {rise }} /(S / N)$. This term can be therefore minimised by increasing $\mathrm{S}$, which can be obtained with an internal gain in the device. This can be reached with Low Gain Avalanche Detectors [5], a diode produced with a thin $\mathrm{p}+$ layer just below the $\mathrm{n}+$ electrode, as shown in figure 3. This device allows moderate gains, 5-20, with local values of the electric field up to $300 \mathrm{kV} / \mathrm{cm}$. Ultra Fast Silicon Detectors $[4,6]$ are LGAD detectors designed to optimise all the relevant parameters of eq. (2.1) in order to minimise $\sigma_{t}$. Besides the choice of an LGAD detector to increase the signal, we can improve $\sigma_{\text {Jitter }}$ by decreasing $t_{\text {rise }}$. This can be accomplished 


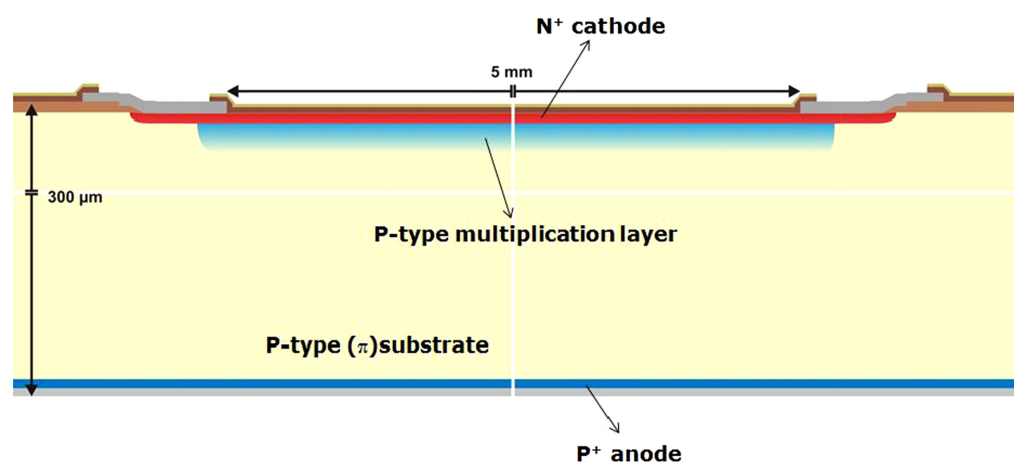

Figure 3. Schematic view of the LGAD pad design, as originally proposed by CNM [5]. Reprinted from [5], Copyright (2014), with permission from Elsevier.

by thinning the detector (tipically to $50-80 \mu \mathrm{m}$ ). A thinner LGAD will produce signals of the same amplitude of a thicker sensor, but with a lower $t_{\text {rise }}$.

Landau fluctuations contribute to $\sigma_{t}$ both by generating Time Walk time spread and by creating a distortion of the signal ( $\left.\sigma_{\text {Landau Noise }}\right)$. Whereas the first contribution can be minimised to a negligible amount by ad hoc design of the FE electronics, the latter is a fixed contribution which cannot be reduced below the critical value of $\sim 20 \mathrm{ps}$, how can be estimated by measuring $\sigma_{\text {Jitter }}^{2}[11]$.

The term $\sigma_{\text {Distorsion }}$ is generated by time fluctuations due to the inhomogeneities of the electric field $\mathrm{E}$ in sensor, essentially related to the geometry of the electrodes. The signal, as described by the Ramo's theorem [7], $i(t)=-q \vec{v} \cdot \overrightarrow{E_{w}}$, in order to minimise the shape fluctuations, needs uniform $\vec{v}$ in the whole sensor volume whilst $\overrightarrow{E_{w}}$ should not vary along the sensor pitch. The first can be obtained by saturating the drift velocity, which occurs for values of the electric filed $E \geqslant 30 \mathrm{kV} / \mathrm{cm}$, while the latter is reached by using wide strips where the strip width has the same dimensions of the strip pitch.

Finally the contribution due to TDC discretization $\left(\sigma_{\mathrm{TDC}}\right)$, which goes as $\mathrm{TDC}_{\mathrm{BIN}} / \sqrt{12}$ can be easily made small by choosing the suitable TDC bin.

\subsection{Front-end and read-out electronics}

Preamplifiers for timing applications belong to two major classes: Broad Band Current amplifiers and Charge Sensitive Amplifiers [8]. Whereas BBA have higher slew rate, and higher noise, little shaping (and therefore more sensitive to Landau bumps), and are more power consuming, CSA have lower slew rate and lower noise, a better signal smoothing given by the signal shaping and low power consumption. The interplay of these characteristics has to be carefully evaluated in the optimization of the time resolution. The time measuring circuit, which stands at the output stage of the preamplifier must determine the time of arrival of the track with the best possible correction for the time walk. This could be ideally achieved with a Multi Sampling circuit, but this option has not been considered since it is not easy to implement it in large scale systems, as needed in HEP experiments. Time is then determined and time walk corrected with Time over Threshold (ToT) or Constant Fraction Discriminator (CFD) circuits. 


\subsection{Beam test results}

The UFSD time resolution was measured on a beam test with pions of $180 \mathrm{GeV} / \mathrm{c}$ momentum at CERN, using 3 UFSD manufactured by CNM within a RD50 Common Project [9], plus a SiPM mounted on a quartz bar for timing crosscheck. The detectors were produced on 4" Silicon-onInsulator wafers with a $45 \mu \mathrm{m}$ thick high resistivity float zone (FZ) active layer on top of a $1 \mu \mathrm{m}$ buried oxide and a $300 \mu \mathrm{m}$ support wafer. The detectors used in the test comprise a single pad structure $1.7 \mathrm{~mm}^{2}$. Three sets of wafers were produced, identical in the mask design but with a different $\mathrm{p}+$ dose of the gain layer to optimise the charge multiplication (only the intermediate gain implant dose were used in the test beam). Time resolutions were determined at $\mathrm{V}_{\text {Bias }}=200 \mathrm{~V}$ and $\mathrm{V}_{\text {Bias }}=230 \mathrm{~V}$, corresponding to Gain values of 20 and 25, using a Constant Fraction Discriminator emulator on data collected with a multisampling digital scope. As shown in figure 4 UFSD can reach a timing resolution of $34 \mathrm{ps}$ at a bias voltage of $200 \mathrm{~V}$ and $27 \mathrm{ps}$ at $230 \mathrm{~V}$. The time resolution of a single UFSD is measured to decrease with increased gain $\mathrm{M}$ like $\mathrm{M}^{-0.36}$. The result is in excellent agreement with the UFSD simulation program Weightfield2 [10].
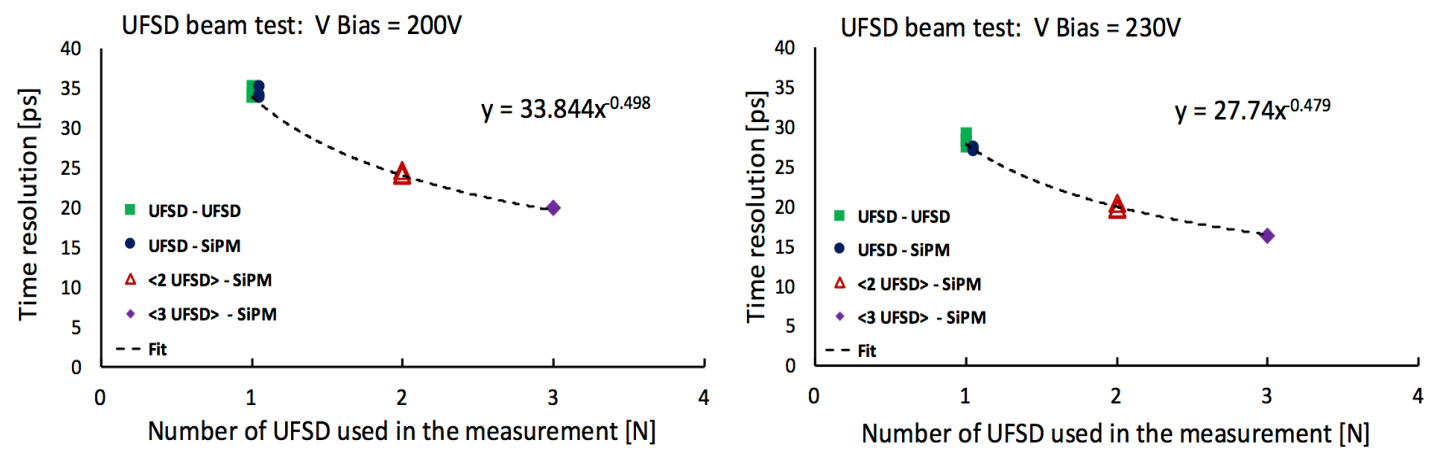

Figure 4. Timing resolution for: single UFSD $(\mathrm{N}=1)$ obtained from the $\mathrm{UFSD}_{i}-\mathrm{SiPM}_{\mathrm{P}}$ and $\mathrm{UFSD}_{i}-\mathrm{UFSD}_{j}$ $(i, j=1,3)$ time differences. The sigma of each measurement is $\sim 10 \%$ of the value, and it is consistent with the scatter of the 6 data points at $\mathrm{N}=1$; averaged pairs of UFSD $(\mathrm{N}=2)$ and average of 3 UFSD $(\mathrm{N}=3)$ for bias voltages of (left) $200 \mathrm{~V}$ and (right) $230 \mathrm{~V}$ [9]. Reproduced from [9]. CC BY 4.0.

Similar results have been obtained using $50 \mu \mathrm{m}$ thick UFSD $1.3 \mathrm{~mm}$ single pad manufactured at Hamamatsu Photonik [11] (see section 3). Time resolution has been determined at various temperatures, and its dependence on gain measured, as shown in figure 5. With a CFD set at 20\%, time resolution is $\sim 30 \mathrm{ps}$. For higher CFD values time jitter improves, but time resolution remains constant being limited by Landau fluctuations (with very moderate temperature dependence).

\section{UFSD radiation tolerance}

Tracking in High Luminosity HEP experiments requires good performance of the sensors at high radiation levels. At High Luminosity LHC timing detectors are required to operate efficiently up to fluences of $5 \cdot 10^{15} \mathrm{n}_{\mathrm{eq}} / \mathrm{cm}^{2}$. LGAD detectors suffer both radiation damage which is typical of silicon sensors with no gain, plus radiation effects affecting directly the performance of the gain layer. Its performance under radiation effects have been studied within the RD50 CERN collaboration [12]. In silicon detectors with no gain the main damage is to the bulk of the sensor caused by the non-ionizing energy loss (NIEL). All bulk effects depend critically on the thickness 


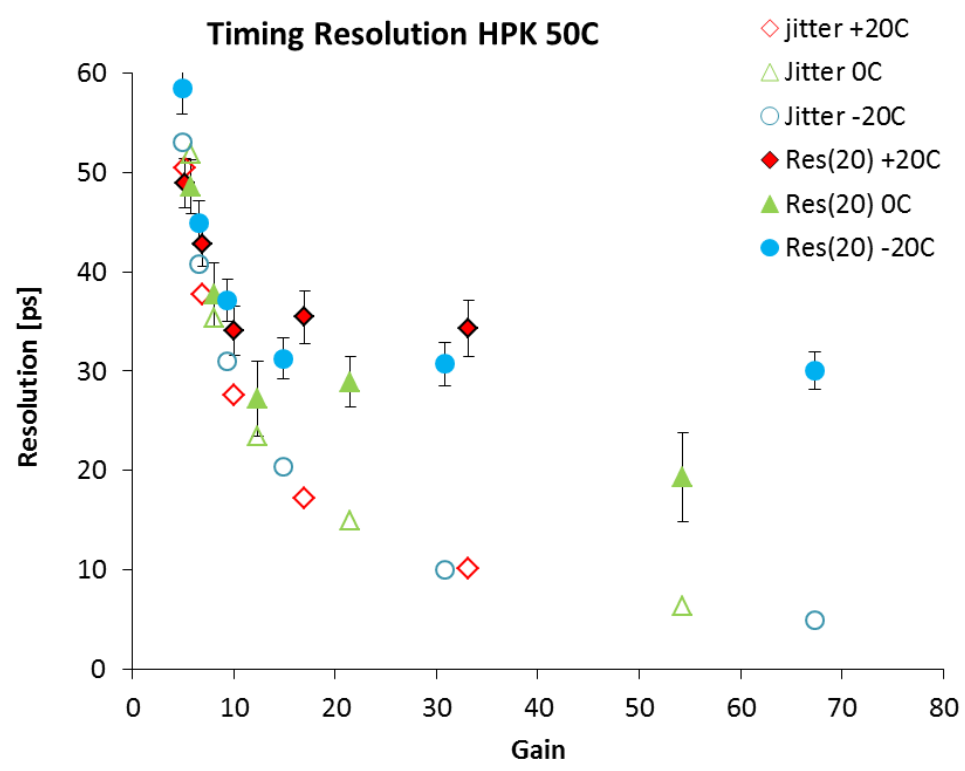

Figure 5. Time Resolution vs Gain of HPK LGAD $50 \mu \mathrm{m} 1.3 \mathrm{~mm}$ single pad sensor at three different temperatures. A Landau contribution of $\sim 20 \mathrm{ps,}$, at high gain, as described in section 2 , can be evaluated as the difference between the measured time resolution (full dots) using a beta source, and the jitter time resolution (empty dots), as measured with few picoseconds 1064nm laser shots [11]. Reproduced with permission from [11].

of the sensor and are proportional to the fluence. Radiation damage is known to cause three main effects: (i) leakage current increase, (ii) changes in doping concentration, and (iii) decrease of charge collection efficiency. The effective doping concentration increases with radiation, and so does the full depletion voltage $\mathrm{V}_{\mathrm{FD}}$ and the breakdown voltage $\mathrm{V}_{\mathrm{BD}}$. Finally, the probability of charge trapping during the drift increases with fluence. It is determined by the trapping time, which for the target fluence and the maximum drift velocity corresponds to a distance of about $50 \mu \mathrm{m}$ [14]. Thus at large fluences only sensors of up to that thickness will collect a majority of the charges created, which happens to be the optimised thickness also to improve the time resolution, as shown in section 2. LGAD sensors add two more specific effects of radiation ageing [15]. The first is an increase of the leakage current due to the gain layer. The leakage current generates more power consumption, which needs carefully designed cooling, and an increase of the shot noise. Since the shot noise rises faster than the signal as a function of the gain, this recommends the use of low gain sensors, kept at low temperatures. Another radiation effect which affects LGAD is the acceptor removal, which is a loss of gain as a function of the fluence in the form $N(\Phi)=N_{0} e^{a \Phi}$. The effect of radiation is the displacement of $B$ atoms which become interstitial, therefore reducing the effective doping concentration.

An irradiation campaign with fluences of $1 \cdot 10^{14}, 3 \cdot 10^{14}, 6 \cdot 10^{14}, 1 \cdot 10^{15}, 3 \cdot 10^{15}$ and $6 \cdot 10^{15} \mathrm{n}_{\mathrm{eq}} / \mathrm{cm}^{2}$ has been completed on $50 \mu \mathrm{m}$ thick UFSD, with a $1.0 \mathrm{~mm}$ diameter active area, produced by Hamamatsu Photonics (HPK), Japan, with pre-radiation internal gain in the range 5-70 depending on the bias voltage [13]. As shown in figure 6a the bias required to reach a certain gain value increases with increased fluence due to the acceptor removal mechanism: as the gain layer becomes less doped and generates a weaker electric field, the external bias voltage needs to be 
increased to compensate for this loss. The simulation program Weightfield2 [10] incorporates the parametrization of the measured acceptor removal and predicts that when a bias value of about $600-650 \mathrm{~V}$ is reached, the electric field in the bulk becomes high enough to generate multiplication also in the sensor bulk, moving the location of the multiplication mechanism from the gain layer into the bulk. For highly irradiated UFSD no strong temperature dependence is observed. The capability of Weightfield2 of reproducing the signal shape in highly irradiated sensors is shown in figure $6 \mathrm{~b}$ where it is also shown another peculiar behaviour of UFSD under irradiation, that is the decrease of the signal rise-time w.r.t. fluence. At $6 \cdot 10^{15} \mathrm{n}_{\mathrm{eq}} / \mathrm{cm}^{2}$ the initial electrons and the gain electrons make the pulse front steeper and trapping reduces the pulse tail. Shorter rise times occurs at high fluences, when the gain layer makes only a small contribution. The time resolution as a function of the gain for different neutron fluences is shown in figure 7, where it can be seen how the decrease of the rise-time at small gains observed at high fluences recovers the loss of time resolution due to the gain layer depletion. At low gain time resolution of highly irradiated samples is even better than for low irradiated samples. This is due to the fact that below a fluence of $1 \cdot 10^{15} \mathrm{n}_{\mathrm{eq}} / \mathrm{cm}^{2}$ the multiplication occurs mainly in the gain layer, while for higher fluences, with smaller gains but with much higher depletion voltages, the multiplication happens everywhere in the bulk, allowing for the multiplication process to start earlier.
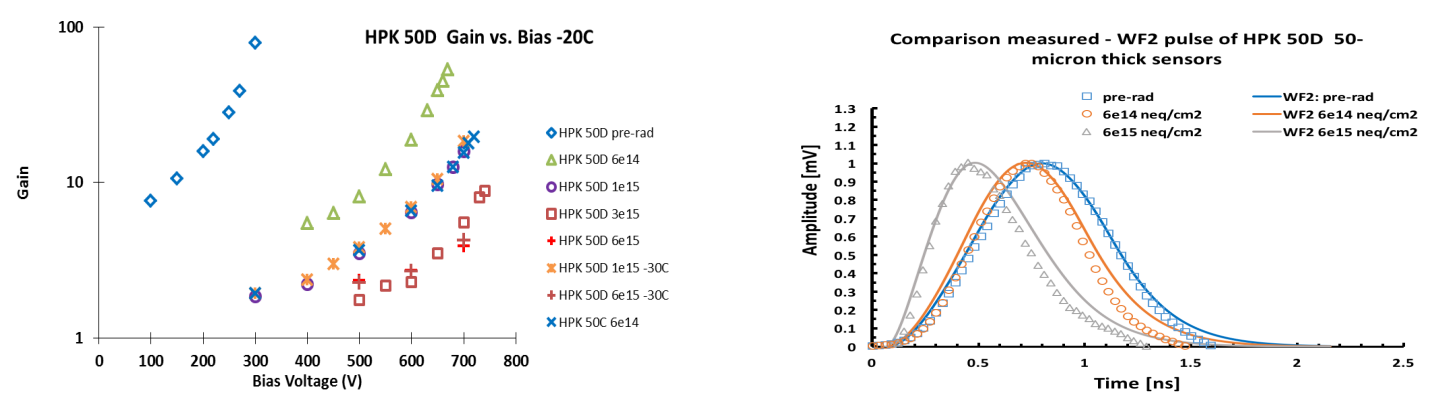

Figure 6. a) Gain as function of $\mathrm{V}_{\mathrm{Bias}}$ for different irradiation profiles, at $-20^{\circ} \mathrm{C}$ and $-30^{\circ} \mathrm{C}$. Letters $\mathrm{C}$ and D indicate sensors with different gain. b) Comparison of data and WF2 simulation program for different irradiation profiles [13]. Reproduced with permission from [13].

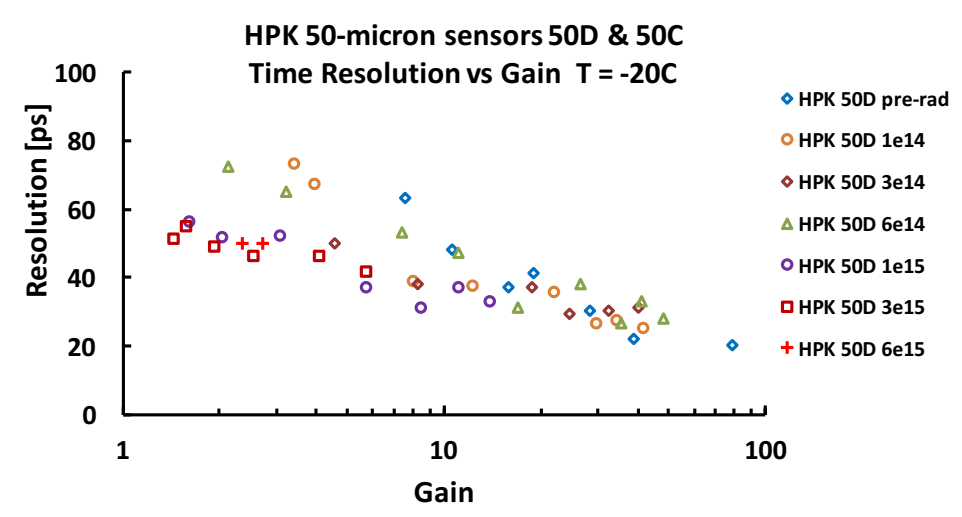

Figure 7. Time Resolution as a function of Gain for different neutron fluences [13]. Reproduced with permission from [13]. 


\section{New FBK production}

In spring 2017 we completed a second UFSD production run with Fondazione Bruno Kessler (FBK, Trento, Italy). The aim of this run is the fabrication of $50 \mu \mathrm{m}$ thick detector (FBK run I was a $300 \mu \mathrm{m}$ sensor production for the UFSD-LGAD process validation), with different acceptors in different gain splits, at two different diffusion temperatures, and many different structures (single pads, multistrips and pixel). The batch is produced with different doping of the gain layer with splits of Carbon co-implantation, both with Boron and Gallium acceptor dopants in order to enhance and test radiation tolerance. The total test production consists of 19 wafers: 10 with B at two different diffusion temperatures, 5 different gain splits, with and without $C$ at two different concentrations, and 9 wafers with Ga with the same splits of B samples. The use of $\mathrm{C}$ and Ga dopants has been proposed within the RD50 collaboration in order to mitigate the disappearance of the gain layer due to radiation damage. Carbon reduces the concentration of interstitials available for capturing $\mathrm{B}$ atoms during irradiation [16], and Gallium replacing B reduces the formation of the acceptor-interstitial [20]. Each wafer contains several structures (see figure 8), single pads, multipad structures, strip structures for CT-PPS detector, microstrip detectors (strip pitch $215 \mu \mathrm{m}$ and 4 different strip lenght, designed for medical physics applications [21]), high granularity multipixel (1800 pixel $300 \times 300 \mu \mathrm{m})$ designed to be bump-bonded to the NA62 TDCpix FE VLSI chip [1].

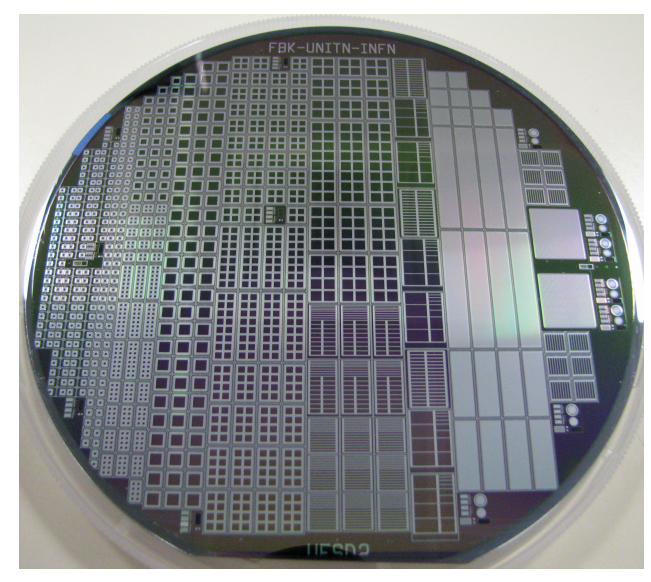

Figure 8. II FBK $50 \mu \mathrm{m}$ thick production, with different single pad, multi-pad, strip, microstrip and pixel detectors.

The first production batch has been tested in the INFN laboratory in Torino and IV and CV curves measured. Figure 9 shows the leakage current vs bias voltage. $\mathrm{C}$ co-implant has the effect of increasing the leakage current both in the Ga and in the B implant test structures (the $\mathrm{C} \mathrm{High}$ implant, which correspond to 10 times the Low implant saturates the device, whereas the Low increases the leakage current more in the B sensors w.r.t. the Ga ones). The observed increase of dark current in $\mathrm{C}$ samples however maintains the same $\mathrm{V}$ dependence observed in samples without $\mathrm{C}$, indicating that the electric internal properties of the device remain unchanged. The IV measurement shows the typical behaviour previously observed in LGAD detectors. Below $20 \mathrm{~V}$ only surface current is visible, between 20 and $30 \mathrm{~V}$ there is a skip which correspond to the full depletion of the gain layer. At higher voltages we observe an exponential dependence due to 
the increase of the gain with increasing electric field E. Breakdown voltage occurs at comparable values, but the Low $\mathrm{C}$ structures have higher $\mathrm{V}_{\mathrm{BD}}$ w.r.t. structures without $\mathrm{C}$, both in $\mathrm{Ga}$ and $\mathrm{B}$ doped sensors. The capacitance drop in the CV curves, as shown in figure 10 indicate that in $\mathrm{Ga}$ structures gain layer full depletion and onset of bulk depletion occurs at $\mathrm{V}_{\text {Bias }}$ slightly higher than with $\mathrm{B}$, with $\mathrm{C}$ having the effect, both in $\mathrm{Ga}$ and $\mathrm{B}$, to lower the gain layer depletion point, consistent with the property of C-coimplantation to affect the B and probably Ga activation, as qualitatively described in ref. [16]. Good uniformity is observed on the various structures tested on wafer prior the final cutting. Detectors are currently being sent to the RD50 colleagues of Jozef Stefan Institute of Ljubljana for the neutron irradiation campaign.
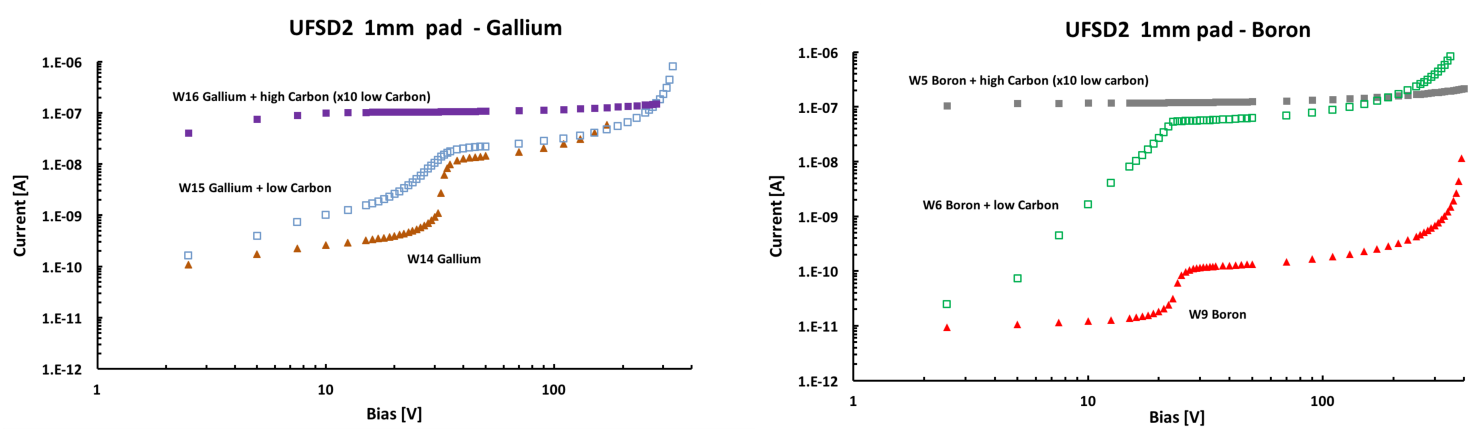

Figure 9. Leakage Current vs $\mathrm{V}_{\mathrm{Bias}}$ measurements on UFSD2 run with $\mathrm{Ga}, \mathrm{Ga}+\mathrm{C}, \mathrm{B}, \mathrm{B}+\mathrm{C}$. $\mathrm{C}$ co-implant comes in two doses, called $\mathrm{Hi}$ and $\mathrm{Lo}$, where $\mathrm{Hi}=10 \mathrm{xLo}$.
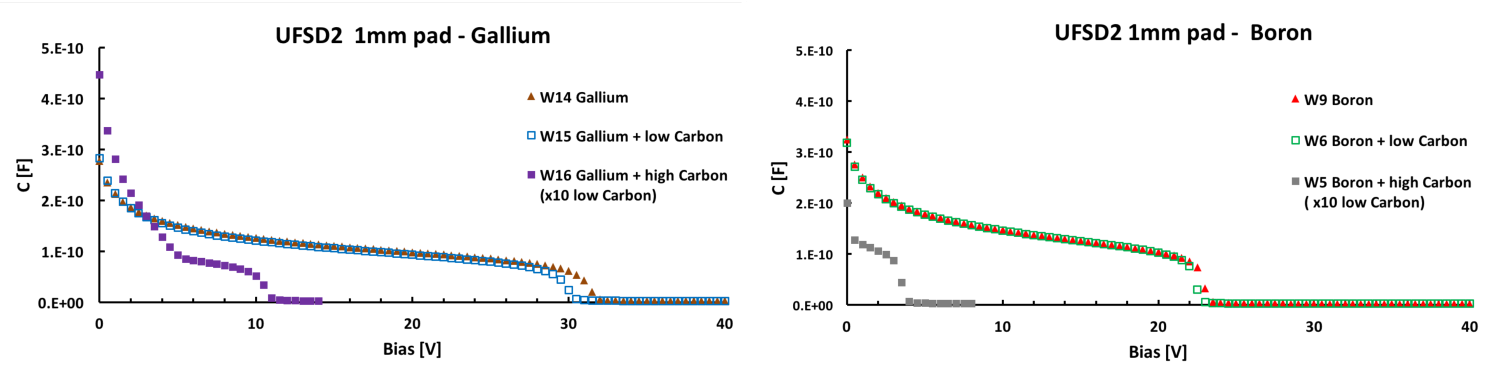

Figure 10. CV Curves for a) Ga, Ga + Low C and Ga + High C and b) CV curves for B, B + Low C and B + High C.

\section{Perspectives on UFSD}

UFSD are currently installed in a HEP experiment, the CMS-Totem Precision Proton Spectrometer (CT-PPS) [17], a detector for the tracking of the surviving scattered protons on both sides of CMS in standard LHC running conditions, using LHC magnets to measure the proton momentum. Tracking and timing detectors are placed at $\sim 220 \mathrm{~m}$ from CMS inside Roman Pots. Proton tracking is performed with 3D silicon pixel detectors and silicon strips, while timing, for bunch crossing identification, is determined with a diamond and an UFSD plane [18]. The detector (see figure 11) is a $45 \mu \mathrm{m}$ thick detector produced by CNM (although, as described in section 4 we have now 
also several FBK II run samples of these detectors), and is readout on $80.5 \times 6 \mathrm{~mm}^{2}+41 \times 3 \mathrm{~mm}^{2}$ custom hybrid board with Surface Mounted Broad Band preamplifiers [19]. The CT-PPS apparatus is currently in its commissioning phase.

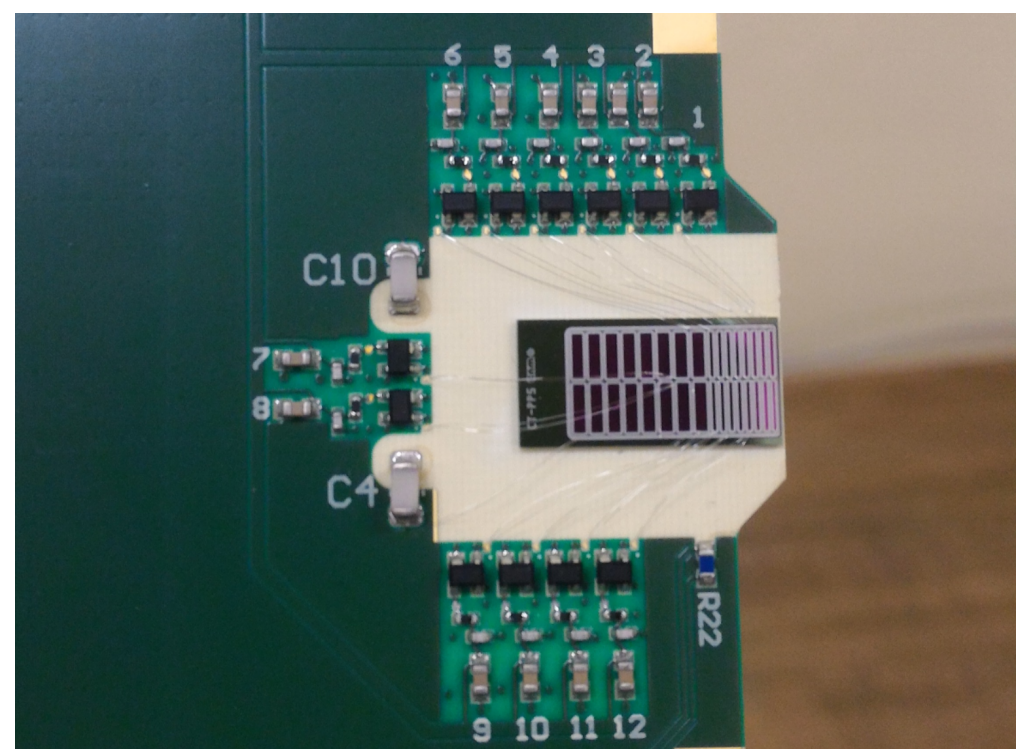

Figure 11. UFSD for timing placed in roman-pots of the CT-PPS detector.

ATLAS and CMS experiments, facing the challenge of the large pile-up expected at HLLHC, are evaluating the possibility to improve pile-up tracks rejection by inserting a Timing Layer made of UFSD between the tracking and the calorimeter sections [3]. In the case of CMS, the timing layer would consist of $\sim 10 \mathrm{~m}^{2}$ to be placed in the endcap region with $1.45 \leqslant \eta \leqslant 3$. The detector specifications require an overall occupancy $\leqslant 1 \%$, a pad surface area of limited dimensions $\left(\sim 3 \mathrm{~mm}^{2}\right)$, to stand the leakage current increasing with the estimated fluence of up to $\sim 10^{15} \mathrm{n}_{\mathrm{eq}} / \mathrm{cm}^{2}$. The gain should be moderate ( $\mathrm{G} \leqslant 20)$, to keep the noise contribution as low as possible. Detailed simulation studies are ongoing to design the detector (doping profiles to optimise the field factor) and the associated electronics.

UFSD applications are currently being pursued also in medical physics, for the monitoring of therapeutical proton beams. An UFSD strip detector has been designed in the II FBK run in order both to directly count individual protons at high rates thus measuring the beam profiles in two orthogonal directions, and to measure the beam energy with time of flight techniques, using a telescope of two UFSD sensors segmented in a minimum number of pads. Results on preliminary tests have been presented within the present conference in [21].

\section{Conclusions}

Ultra Fast Silicon Detectors are quickly becoming a mature technology to be used in high granularity timing sensitive applications, and its behaviour is well understood and reproduced by simulation programs (Weightfield2 and TCAD). The goal time resolution of $\sim 30 \mathrm{ps}$ on single pad has been achieved on beam tests performed on detectors produced by different manufacturers. Results after 
exposure to NIEL at $610^{15} \mathrm{n}_{\mathrm{eq}} / \mathrm{cm}^{2}$, levels to be considered in HL-LHC, look very promising, and further improvements, investigated with new implants of $\mathrm{Ga}$ and $\mathrm{C}$ are currently under test (II FBK run). The interest and soundness of the LGAD technology is well proven by the rapidly spreading commitment of different manufacturers, as CNM, FBK, HPK and more recently Micron Semiconductos ltd [22].

\section{Acknowledgments}

We acknowledge the fundamental contributions coming from the discussions, and active collaboration of the RD50 colleagues. Part of this work has been financed by the European Union's Horizon 2020 Research and Innovation funding program, under Grant Agreement no. 654168 (AIDA-2020) and Grant Agreement no. 669529 (ERC UFSD669529), and by the Italian Ministero degli Affari Esteri and INFN Gruppo V.

\section{References}

[1] G. Aglieri Rinella et al., The NA62 GigaTracker, Nucl. Instrum. Meth. A 845 (2017) 147.

[2] G. Apollinari et al., High-Luminosity Large Hadron Collider (HL-LHC): Preliminary Design Report, CERN-2015-005.

[3] L. Gray, 4D trackers (space + time informations), in Connecting the Dots/Intelligent Tracker 2017, LAL-Orsay, France, 6-9 March 2017.

[4] H.F.W. Sadrozinski, A. Seiden and N. Cartiglia, 4-Dimensional Tracking with Ultra-Fast Silicon Detectors, arXiv: 1704.08666.

[5] G. Pellegrini et al., Technology developments and first measurements of Low Gain Avalanche Detectors (LGAD) for high energy physics applications, Nucl. Instrum. Meth. A 765 (2014) 12.

[6] H.F.W. Sadrozinski et al., Ultra-fast silicon detectors (UFSD), Nucl. Instrum. Meth. A 831 (2016) 18.

[7] S. Ramo, Currents Induced by Electron Motion, Proc. IRE 27 (1939) 584.

[8] N. Cartiglia et al., Design optimization of ultra-fast silicon detectors, Nucl. Instrum. Meth. A 796 (2015) 141.

[9] N. Cartiglia et al., Beam test results of a 16 ps timing system based on ultra-fast silicon detectors, Nucl. Instrum. Meth. A 850 (2017) 83 [arXiv: 1608.08681].

[10] F. Cenna et al., Weightfield2: A fast simulator for silicon and diamond solid state detector, Nucl. Instrum. Meth. A 796 (2015) 149.

[11] H. Sadrozinski et al., Timing Measurements on Ultra-Fast Silicon Detectors, in 12th Trento Workshop on Advanced Silicon Radiation Detectors, Trento, Italy, 20 February 2017.

[12] RD50 collaboration, http://rd50.web.cern.ch/rd50/.

[13] Z. Galloway et al., Properties of HPK UFSD after neutron irradiation up to $6 \cdot 10^{15} \mathrm{n}_{e q} / \mathrm{cm}^{2}$, arXiv: 1707.04961.

[14] G. Kramberger, V. Cindro, I. Mandic, M. Mikuz and M. Zavrtanik, Effective trapping time of electrons and holes in different silicon materials irradiated with neutrons, protons and pions, Nucl. Instrum. Meth. A 481 (2002) 297. 
[15] G. Kramberger et al., Radiation effects in Low Gain Avalanche Detectors after hadron irradiations, 2015 JINST 10 P07006.

[16] Y. Shimizu et al., Impact of carbon co-implantation on boron distribution and activation in silicon studied by atom probe tomography and spreading resistance measurements, Jpn. J. Appl. Phys. 55 (2016) 026501.

[17] M. Albrow et al., CMS-TOTEM Precision Proton Spectrometer, CERN-LHCC-2014-021.

[18] V. Sola, The CT-PPS Project, in Low x 2017, Bisceglie, Italy, 12-18 June 2017.

[19] TOTEM collaboration, G. Antchev et al., Diamond Detectors for the TOTEM Timing Upgrade, 2017 JINST 12 P03007 [arXiv: 1701.05227].

[20] A. Khan et al., Strategies for improving radiation tolerance of Si space solar cells, Solar Energy Mater. Solar Cells 75 (2003) 271.

[21] A. Vignati, Innovative thin silicon detectors for monitoring of therapeutic proton beams: preliminary beam tests, in $11^{\text {th }}$ International Conference on Position Sensitive Detectors, The Open University, Milton Keynes, U.K., 3-8 September 2017.

[22] R. Bates, Simulation and characterisation of low gain avalanche detector for particle physics and synchrotron applications, in $11^{\text {th }}$ International Conference on Position Sensitive Detectors, The Open University, Milton Keynes, U.K., 3-8 September 2017. 